\title{
Tramadol Induced Seizures, A potential Silent Hazard of the Emergency Toxicology in Iran
}

\section{Behzadnia MJ*}

Department of Emergency Medicine, Baqiyatallah University of Medical Sciences, Iran

*Corresponding author: Mohammad Javad Behzadnia, Trauma Research Center, Department of Emergency Medicine, Baqiyatallah University of Medical Sciences,

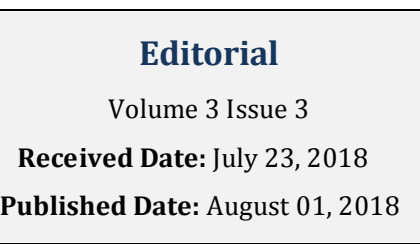

Tehran - Iran IR. Tel: +982181262012; Email: behzadnia@bmsu.ac.ir

\begin{abstract}
Tramadol is a synthetic analgesic drug that has a potential risk of addiction. Due to its common illegal abuse, tramadol induced seizures are becoming more prevalent. Although these seizures are self-limited in most of the cases, however, the subsequent complications such as trauma and addiction are still the main problems. In this study, we provide a brief introduction of this important topic in Iran with emphasis on the proper medical use of this drug.
\end{abstract}

Keywords: Tramadol; Seizure; Toxicity; Side Effects

\section{Abbreviations: GABA: Gamma-Amino butyric Acid}

\section{Editorial}

Tramadol is used as an analgesic for mild to severe pains. Due to its opioid effects, it is routinely abused by opium addicts.This problem is a general concern worldwide.Tramadol overdose is reported as $1.2 \%$ of all poisonings in Iran of which $65 \%$ were of tramadol only [1].

In order to have effective management, we mention the clinical presentation and prominent complications, based on the most recent studies from Iran. Several studies have reported different side effects of tramadolamong which neurological, psychological and gastrointestinal symptoms are considered as the most common adverse effects [2,3].

As a synthetic analgesic drug, tramadol could lead to seizures even in therapeutic doses. Besides, due to its various effects on GABA and serotonin receptors, its exact mechanism of seizure is not still recognized [4]. Suppression of GABA ergic inhibitory effects and enhancement of neural excitability of tramadol are the two main reasons that may lead to seizures. Tramadol induced seizures are not dose dependent. Several patients have experienced seizures only after taking a single pill. While the suicide by drugs is more prevalent in females, tramadol induced seizures are more seen in males, especially in non-suicidal cases and within a mean age range of 20-25 years old. 6,9,3. Male predominance and the early age onset of seizure may be due to high level of some gender and age specific metabolite concentration [5].

An interesting study reported the prevalence of seizures in acute single dose agent exposure. Tramadol induced seizures were placed after mefenamic acid and few anti-depressant drugs in Iran [6]. Most of the reported mortalities were due to tramadol abuse that had not been prescribed by a physician. In a survey, tramadol mortality was reported due to the risk factors such as depression, seizures and addiction [7]. While magnesium sulfate is recommended for seizure prevention and 


\section{Advances in Clinical Toxicology}

arrhythmia, it seems that tramadol induced seizures even in the case of recurrent history may not need anticonvulsant therapy, as studies have reported no sequelae in these patients [8-10].

Seizure induced trauma is another importantpoint. Recent studies showed that about $1 \%$ of serious head traumas are related to tramadol induced seizures in Iran. 13 Recurrent shoulder dis location was reported in about $1: 4$ of the patients with tramadol induced seizures in which most had anterior shoulder dislocation [11]. Tramadol induced seizures may be related to sodium and potassium channel blockade effects as seen in various patterns of EKG manifestations. However, these patterns are not specific for tramadol effects and showed no significant outcome [12]. Among the clinical presentations, myosis, mydriasis and tachycardia are considered as symptoms associated with the risks for seizures $[1,8]$.

In tramadol overdose, neurological manifestations such as seizures and unconsciousness are more prominent. Tramadol induced seizuresarenot dose dependent but the most common dose in which seizure occurs is 500-1000 mg. Although a high dose consumption correlates with a higher seizure occurrence, however, blood level is not necessarily associated with convulsions [13,14]. Based on the current studies, majority of cardiological and neurological manifestations are transient and appear within the first 24 hours of ingestion. 15 Most of the seizures may even occur within the first 3 hours in an emergency setting [15]. These presentations are more common among the alcoholics, and anti-depressant and anti-psychiatric drug users $[14,15]$. Now a day, because of its idiosyncratic complications, tramadol is no longer prescribedas the first choice in pain management. It is strongly recommended to select proper patients for tramadol prescription and warning the society about the hazardous side effects of this addictive opioid drug.

\section{References}

1. Tashakori A, Afshari R (2010) Tramadol overdose as a cause of serotonin syndrome: a case series. Clin Toxicol (Phila) 48(4): 337-341.

2. Moulis F, Rousseau V, Abadie D, Masmoudi K, Micallef J, et al. (2017) Serious adverse drug reactions with tramadol reported to the French pharmacovigilance database between 2011 and 2015. Therapie 72(6): 615-624.
3. Tsutaoka BT, Ho RY, Fung SM (2015) Kearney TE Comparative Toxicity of Tapentadol and Tramadol Utilizing Data Reported to the National Poison Data System. Ann Pharmacother 49(12): 1311-1316.

4. Bameri B, Shaki F, Ahangar N, Ataee R, Samadi M, et al. (2018) Evidence for the Involvement of the Dopaminergic System in Seizure and Oxidative Damage Induced by Tramadol. Int J Toxicol 37(2): 164-170.

5. Ahmadimanesh M, Shadnia S, Rouini MR, Sheikholeslami B, Nasab AS, et al. (2018) Correlation between plasma concentrations of tramadol and its metabolites and the incidence of seizure in tramadolintoxicated patients. Drug Metab Pers Ther 33(2): 7583.

6. Reichert C, Reichert P, Tschudi MF, Kupferschmidt H, Ceschi A, et al. (2014) Seizures after single-agent overdose with pharmaceutical drugs: analysis of cases reported to a poison center. Clin Toxicol (Phila) 52(6): 629-634.

7. Randall C, Crane J (2014) Tramadol deaths in Northern Ireland: a review of cases from 1996 to 2012. J Forensic Leg Med 23: 32-36.

8. Afshari R, Ghooshkhanehee H (2009) Tramadol overdose induced seizure, dramatic rise of CPK and acute renal failure. J Pak Med Assoc 59(3): 178.

9. Najari F, Alizadeh-Ghamsari A, Vahabzadeh $M$, Dadpour B, Mousavi SR, et al. (2017) A Study of the Importance of Clonus Symptoms in Patients with Tramadol Poisoning. J Toxicol 2017: 4.

10. Shadnia S, Brent J, Fatemi MK, Hafezi P, Soltaninejad $\mathrm{K}$, et al. (2012) Recurrent seizures in tramadol intoxication: implications for therapy based on 100 patients. Basic Clin Pharmacol Toxicol 111(2): 133136.

11. Amroodi NM, Iri A, Akhoondi S (2015) The definition of recurrent shoulder dislocation in tramadol induced seizure patients. Med J Islam Repub Iran 29: 298.

12. Emamhadi M, Zadeh SH, Nikniya M, Zamani N, Dart RC (2012) Electrocardiographic manifestations of tramadol toxicity with special reference to their ability for prediction of seizures. Am J Emerg Med 30(8): 1481-1485.

13. Taghaddosinejad F, Mehrpour O, Afshari R, Seghatoleslami A, Abdollahi M, et al. (2011) Factors 
related to seizure in tramadol poisoning and its blood concentration. J Med Toxicol 7(3): 183-188.

14. Talaie H, Panahandeh R, Fayaznouri M, Asadi Z, Abdollahi M (2009) Dose-independent occurrence of seizure with tramadol. J Med Toxicol 5(2): 63-67.
15. Boostani R, Derakhshan S (2012) Tramadol induced seizure: A 3-year study. Caspian J Intern Med 3(3): 484-487.

\section{(c) (1)}

